\title{
Auditory and visual impairments in patients with blast-related traumatic brain injury: Effect of dual sensory impairment on Functional Independence Measure
}

\author{
Henry L. Lew, MD, PhD; ${ }^{1-4}$ Donn W. Garvert, BA; ${ }^{5}$ Terri K. Pogoda, PhD; ${ }^{4,6}$ Pei-Te Hsu, MD; ${ }^{1-2,4}$ Jennifer M. \\ Devine, MD; ${ }^{1}$ Daniel K. White, PT, ScD; ${ }^{7}$ Paula J. Myers, PhD; ${ }^{8}$ Gregory L. Goodrich, $\mathbf{P h D}{ }^{5 *}$ \\ ${ }^{1}$ Physical Medicine and Rehabilitation Service, Department of Veterans Affairs (VA) Boston Healthcare System, Boston, \\ MA; ${ }^{2}$ Harvard Medical School, Boston, MA; ${ }^{3}$ Defense and Veterans Brain Injury Center, VA Boston Healthcare System, \\ Boston, MA; ${ }^{4}$ Traumatic Brain Injury Center of Excellence, VA Boston Healthcare System, Boston, MA; ${ }^{5}$ VA Palo Alto \\ Health Care System, Palo Alto, CA; ${ }^{6}$ Center for Organization, Leadership and Management Research, VA Boston \\ Healthcare System, Boston, MA; ${ }^{7}$ Boston University Medical Center, Boston, MA; ${ }^{8}$ Audiology Section, James A. Haley \\ Veterans' Hospital, Tampa, FL
}

\begin{abstract}
The frequencies of hearing impairment (HI), vision impairment (VI), or dual (hearing and vision) sensory impairment (DSI) in patients with blast-related traumatic brain injury (TBI) and their effects on functional recovery are not well documented. In this preliminary study of 175 patients admitted to a Polytrauma Rehabilitation Center, we completed hearing and vision examinations and obtained Functional Independence Measure (FIM) scores at admission and discharge for 62 patients with blast-related TBI. We diagnosed HI only, VI only, and DSI in $19 \%, 34 \%$, and $32 \%$ of patients, respectively. Only $15 \%$ of the patients had no sensory impairment in either auditory or visual modality. An analysis of variance showed a group difference for the total and motor FIM scores at discharge $(p<0.04)$. Regression model analyses demonstrated that DSI significantly contributed to reduced gain in total $(t=$ $-2.25)$ and motor $(t=-2.50)$ FIM scores $(p<0.05)$. Understanding the long-term consequences of sensory impairments in the functional recovery of patients with blast-related TBI requires further research.
\end{abstract}

Key words: blast-related, brain injuries, dual sensory impairment, Functional Independence Measure, hearing, outcomes assessment, polytrauma, rehabilitation, sensory disorders, vision.

\section{INTRODUCTION}

Traumatic brain injury (TBI) affects an estimated 1.5 million Americans each year, causing approximately 50,000 deaths and resulting in significant disability and societal cost [1-2]. In the adult civilian population, common causes of TBI include motor vehicle accidents, falls, gunshot wounds, and assaults. Among soldiers returning from Operation Iraqi Freedom (OIF) and Operation Enduring Freedom (OEF), the incidence of TBI is, by

\footnotetext{
Abbreviations: ANOVA $=$ analysis of variance, DSI $=$ dual sensory impairment, FIM = Functional Independence Measure, GCS = Glasgow Coma Scale, $\mathrm{HI}=$ hearing impairment, $\mathrm{HL}=$ hearing loss, ICD-9 = International Classification of Diseases9th Revision, LOC $=$ loss of consciousness, NSI $=$ no sensory impairment, $\mathrm{OEF}=$ Operation Enduring Freedom, OIF = Operation Iraqi Freedom, PRC = Polytrauma Rehabilitation Center, PTA = posttraumatic amnesia, TBI = traumatic brain injury, VA = Department of Veterans Affairs, VI = vision impairment.

*Address all correspondence to Gregory L. Goodrich, PhD; Psychology Service, VA Palo Alto Health Care System, 3801 Miranda Avenue, Palo Alto, CA 94304; 650-493-5000, ext 64385; fax: 650-852-3239. Email: gregory.goodrich@va.gov DOI:10.1682/JRRD.2008.09.0129
} 
some estimates, as high as 15 percent [3]. Thus, TBI has been referred to as a "signature wound" of the Global War on Terrorism [4].

Military servicemembers equipped with advanced body armor designed to protect vital organs are surviving injuries from explosive devices and rocket-propelled grenades that would have been fatal in the past [5]. Blastrelated injuries from explosive devices are estimated to be responsible for 60 percent of TBI in military personnel [6]. As these patients present to Department of Veterans Affairs (VA) Polytrauma Rehabilitation Centers (PRCs), they pose unique and complex rehabilitative challenges for clinicians. Investigating the consequence of blastrelated injuries is timely and necessary, given the influx of wounded veterans with TBI from OIF/OEF [7].

Because of the traumatic nature of TBI, concurrent damage to the auditory system can occur anywhere from the outer ear, middle ear, and inner ear to the auditory cortex, resulting in impaired auditory function [8-11]. Although the current literature suggests hearing impairment (HI) is prone to occur, it does not adequately describe the characteristics of $\mathrm{HI}$ associated with blastrelated TBI [11-12]. Vision impairment (VI) is also commonly noted in patients with blast-related TBI [13-14]. Given the occurrence of both HI and VI in the population with blast-related TBI, a subset of patients with TBI likely experiences impairments to both sensory systems. To date, the literature has not well described the occurrence of this dual sensory impairment (DSI) in TBI. One study of non-TBI VA outpatients reported an overall prevalence of DSI in patients $\geq 65$ years old as high as 7.4 percent; in a subsample of patients $<65$ years old, DSI was not reported [15]. During the rehabilitation process, patients with DSI may encounter more challenges than sensory-intact patients with TBI, especially with tasks requiring visual and/or auditory communication. However, the functional consequences of DSI in persons recovering from TBI are also unknown.

While many TBI studies on the civilian population exist, less is known about OIF/OEF patients who have incurred blast-related TBI [2]. The purpose of this study is twofold. First, we describe the occurrence of documented HI only, VI only, and DSI in OIF/OEF veterans who sustained blast-related TBI and received treatment at a PRC. Second, we determine the effect of HI only, VI only, and DSI on these patients' (1) functional status at admission, (2) functional status at discharge, and (3) functional improvement during rehabilitation as measured by the Functional Independence Measure (FIM). We hypothesized that patients with DSI would have poorer FIM scores at admission and discharge and make fewer functional gains during rehabilitation than patients with a single sensory (auditory or visual) impairment or no sensory impairment (NSI).

\section{METHODS}

\section{Participants}

Data were extracted from comprehensive record reviews of 175 patients with TBI admitted to a PRC between December 2004 and March 2008 (40 months). We included only patients not previously admitted to the PRC for TBI treatment. The sample was divided into four subgroups based on diagnoses of sensory impairments, specifically HI only, VI only, DSI, and NSI (Figure).

\section{Auditory Assessments}

Patients saw the audiology consult service and underwent immittance and pure tone audiometric evaluations. Clinically certified audiologists used the QT1 Quik Tymp Tympanometer (American Electromedics Corp; Amherst, New Hampshire) to determine the integrity of the tympanic membrane and middle ear/ossicular system. [11]. Pure tone thresholds were obtained using the modified HughsonWestlake procedure [16]. Audiologists conducted the examinations in a double-walled, sound-treated booth using a Grason-Stadler GSI 61 clinical audiometer (CareFusion; San Diego, California). Air- and bone-conduction audiometric thresholds were assessed to quantify the peripheral hearing function at different frequencies [17]. For the purposes of this study, we classified HI severity based on the poorest audiometric threshold in the poorer ear at any frequency (across thresholds of 250, 500, 1,000, 2,000, 4,000, and 8,000 Hz): (1) mild hearing loss (HL), 26 to $40 \mathrm{~dB}$; (2) moderate HL, 41 to $60 \mathrm{~dB}$; (3) severe HL, 61 to $90 \mathrm{~dB}$; and (4) profound HL, >90 dB.

\section{Visual Assessments}

The optometric and ophthalmologic teams performed vision assessments. A previous study described specific elements of the vision examination protocol [13]. It consists of vision status self-report before and after injury; distance and near visual acuity clinical measurements; visual field status; binocular vision status; and other vision measures, including reading speed and comprehension assessments. We examined patients at bedside if they had insufficient mobility to travel to the eye clinic for a traditional examination. Visual acuities were assessed 


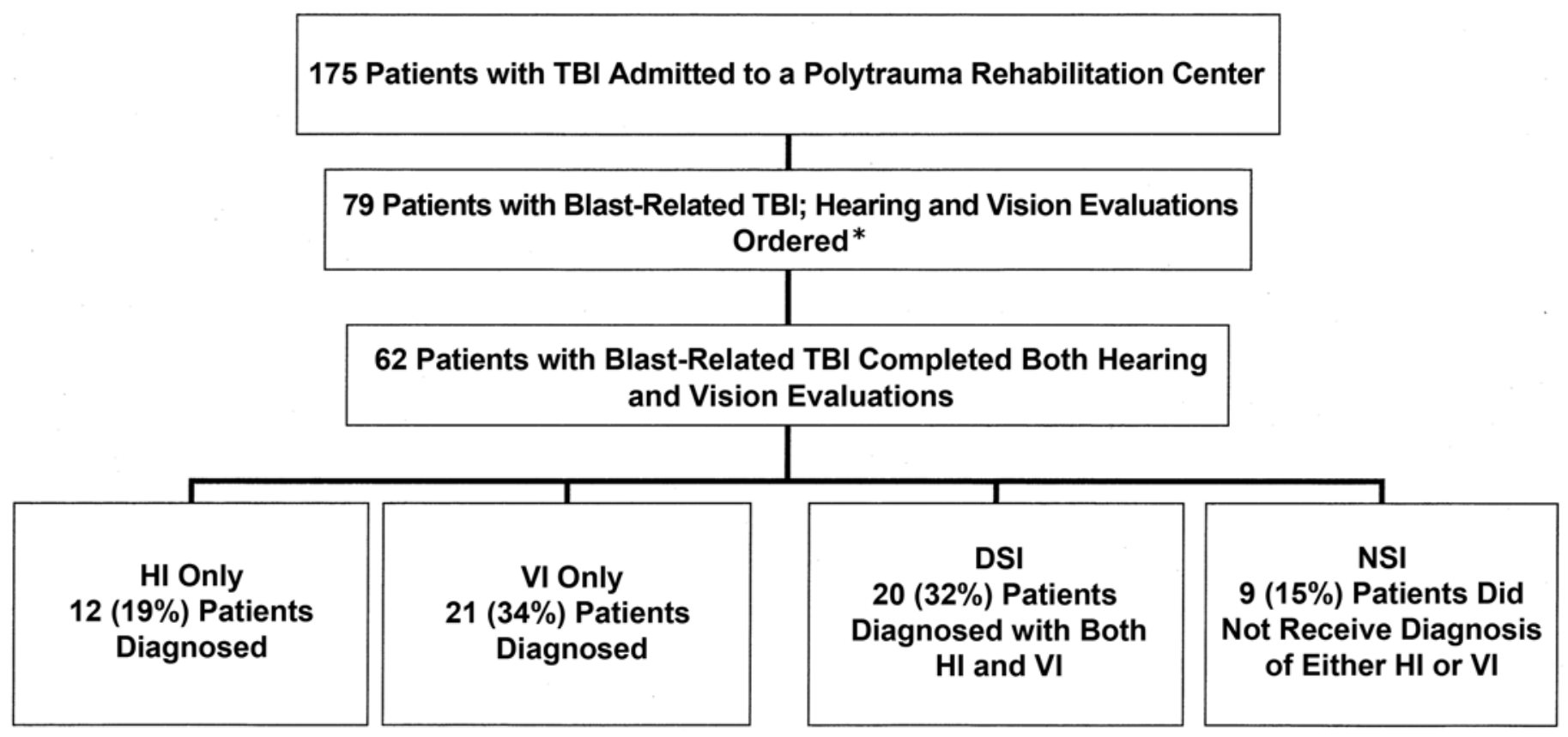

Figure.

Distribution of sensory impairments. * Data unavailable for 17 patients because of missed appointments, short lengths of stay, or unresponsiveness due to severity of injury. DSI = dual sensory impairment, HI = hearing impairment, NSI = no sensory impairment, TBI = traumatic brain injury, $\mathrm{VI}=$ vision impairment.

using the Feinbloom chart (Lighthouse International; New York, New York) at a distance of 10 feet. Acuity cards with single numbers were used if a patient was nonverbal but able to respond to yes/no questions by head nod or finger indication. Optometrists measured near acuities using text, single words, triple digits, or single digits, depending on the patient's abilities. We based visual acuity on bestcorrected refraction of the better eye, depending on the level of cooperation and existing corrections provided by the patient.

For the purposes of this article and to reflect VA eligibility for vision rehabilitation services, the definition of visual acuity impairment was based on the International Classification of Diseases-9th Revision (ICD-9) classification [18]. Normal and near-normal measurements were combined into a single level, defined as visual acuity between 20/20 and 20/63, because patients with these acuities would typically be ineligible for services. Patients with ICD-9 levels of moderate, severe, and profound VI were combined into a single category, defined as visual acuity ranging from $<20 / 63$ (e.g., 20/70) to 20/1,000, because all would be eligible for VA vision rehabilitation services. Patients with hemianopsia comparable with a VI of $\leq 20^{\circ}$ were also considered to have VI and would be eligi- ble for VA vision rehabilitation services. Blindness was classified as a visual acuity of $<20 / 1,000$ or bilateral enucleation, because these patients would receive blind rehabilitation services rather than low-vision rehabilitation services. To encompass the full range of VI reported in this population and to be consistent with current VA guidelines, patients with binocular dysfunction were included in the group with VI.

\section{Functional Assessments}

The FIM was the primary outcome measure used to assess functional status during inpatient rehabilitation. This measure is an 18-item, 7-level ordinal scale that assesses patients' functional independence. It comprises a motor subscale (13 items of self care, mobility, locomotion, and sphincter control) and a cognitive subscale (5 items of communication and social-cognition), with total scores ranging from 18 to 126. A higher score denotes a higher level of functional independence. Though not well-known as being sensitive to changes in cognitive and communicative function, the FIM is a valid measure of functional independence in individuals with TBI [19]. All PRC healthcare providers who administered the FIM were required to pass the credentialing test to ensure the accuracy and uniformity 
of their assessments. The FIM data were stored in the Functional Status Outcomes Database. As a hospital policy, healthcare providers completed FIM scores within 72 hours of patient admission and at discharge.

\section{Traumatic Brain Injury Severity}

TBI severity was categorized as mild, moderate, or severe using accepted clinical diagnostic criteria, including Glasgow Coma Scale (GCS) score at time of acute care presentation, duration of posttraumatic amnesia (PTA), and/or duration of posttraumatic loss of consciousness (LOC). Mild TBI was defined as an initial GCS score of 13 to 15, PTA duration of $<1$ day, or LOC duration of $<1$ hour. Moderate TBI was defined as an initial GCS score of 9 to 12, PTA duration between 1 day and $<1$ week, or LOC duration between 1 and 24 hours. Severe TBI was defined as an initial GCS score of 3 to 8, PTA duration of $>1$ week, or LOC duration of $>24$ hours. Because most patients did not have all three clinical diagnostic criteria available in their medical records, the TBI severity rating was based on the most severe categorization of the GCS score, PTA, or LOC.

\section{Data Collection}

The Stanford University Institutional Review Board and VA Research and Development Committee approved the protocol for this study. We performed a retrospective chart review on all new admissions between December 1, 2004, and March 31, 2008. Data on demographics, hospitalization course, and HI/VI were obtained from the Computerized Patient Record System. Experienced clinical staff who worked with a wide variety of disabilities related to patients with polytrauma documented the medical records. They routinely submitted auditory and visual consultations for patients with blast-related TBI. Appropriate devices were prescribed to facilitate communication between staff and patients with communication deficits. None of the records we reviewed indicated that communication problems interfered with the clinicians' ability to conduct their examinations.

\section{Statistical Analysis}

Occurrence and co-occurrence of $\mathrm{HI}$ and VI was determined after reading reports from the audiology and optometry/ophthalmology consulting services. To evaluate the relationship between various types of sensory impairments and changes in functional status, we employed analysis of variance (ANOVA) for continuous outcomes and fol- lowed up with Tamhane's T2 (equal variances not assumed) post hoc $t$-tests. Dependent variables included FIM (total, motor, and cognitive scores) at admission and discharge and improvement between admission and discharge (admission FIM to discharge FIM). Finally, to evaluate significant predictors of FIM score changes from admission to discharge, separate multiple regression analyses for total, motor, and cognitive FIM scores were performed. Variables simultaneously entered into these models were overall function at admission (total FIM score at admission), sensory impairment status (HI only, VI only, DSI), age, time since injury, and TBI severity (mild, moderate, severe).

\section{RESULTS}

\section{Participant Demographics}

The medical records of 175 patients with TBI who were admitted to a PRC were reviewed. Hearing and vision evaluations were ordered for the 79 patients with blast-related TBI (Figure). Of these patients, 17 did not receive one or both of these evaluations because of missed appointments, short lengths of stay, or unresponsiveness due to severity of injury. The 62 patients who completed both hearing and vision examinations had a mean age of 27.3 years (standard deviation 7.0 years; range: $19-47$ years). The majority were male ( $n=58 ; 93.5 \%$ ), Caucasian ( $n=46 ; 74.2 \%$ ), and the average time since injury was 238.5 days. The Figure shows the flowchart of the population and describes the incidences of HI only, VI only, and DSI diagnoses made by the hearing and vision consulting services during inpatient rehabilitation.

\section{Distribution of Sensory Impairments}

Among the 62 patients who received evaluations by both audiology and optometry/ophthalmology consultations, we diagnosed sensory impairments in 53 (85.5\%) of the cases. We found NSI in 9 (14.5\%) and HI only, VI only, and DSI in 12 (19.4\%), 21 (33.9\%), and 20 (32.3\%) of the patients, respectively.

\section{Relationship Between Sensory Impairment and Functional Independence Measure}

\section{Total, Motor, and Cognitive Functional Independence Measure}

Table 1 shows the total, motor, and cognitive FIM scores at admission and discharge, and the change over 
Table 1.

Total, motor, and cognitive Functional Independence Measure (FIM) score changes (mean \pm standard deviation) over time among 62 veterans with blast-related traumatic brain injury, stratified by sensory impairment group.

\begin{tabular}{|c|c|c|c|c|c|c|c|c|c|}
\hline \multirow{2}{*}{$\begin{array}{c}\text { Sensory } \\
\text { Impairment }\end{array}$} & \multicolumn{3}{|c|}{ Admission } & \multicolumn{3}{|c|}{ Discharge } & \multicolumn{3}{|c|}{ Score Change (Gain) } \\
\hline & Total & Motor & Cognitive & Total $^{*}$ & Motor ${ }^{*}$ & Cognitive & Total & Motor & Cognitive \\
\hline NSI $(n=9)$ & $109.2 \pm 28.1$ & $80.0 \pm 25.5$ & $29.2 \pm 4.3$ & $120.1 \pm 6.0$ & $89.0 \pm 4.6$ & $31.1 \pm 3.1$ & $10.9 \pm 22.9$ & $9.0 \pm 21.0$ & $1.9 \pm 2.8$ \\
\hline VI Only $(n=21)$ & $103.0 \pm 29.3$ & $76.0 \pm 23.6$ & $27.0 \pm 7.0$ & $118.2 \pm 5.7$ & $87.6 \pm 4.6$ & $30.6 \pm 3.2$ & $15.2 \pm 24.6$ & $11.6 \pm 19.8$ & $3.6 \pm 5.4$ \\
\hline DSI $(n=20)$ & $95.3 \pm 30.6$ & $70.1 \pm 25.9$ & $25.3 \pm 7.3$ & $107.7 \pm 19.2$ & $79.7 \pm 15.4$ & $28.1 \pm 6.3$ & $12.4 \pm 16.0$ & $9.6 \pm 14.7$ & $2.8 \pm 2.8$ \\
\hline
\end{tabular}

${ }^{*}$ For total and motor FIM scores at discharge, group with DSI scored marginally lower than group with NSI $(p<0.09)$.

DSI = dual sensory impairment, $\mathrm{HI}$ = hearing impairment, NSI = no sensory impairment, VI = vision impairment.

time, stratified by sensory impairment group. A visual inspection of the mean values shows that the HI only group had lower FIM scores at admission, though this pattern was not statistically significant $(F(3,58)=0.69, p>$ $0.05)$. Overall, the patient groups did not differ in any of their FIM scores at admission. At discharge, however, ANOVA showed the groups differed in total FIM scores $(F(3,58)=3.05, p<0.04)$ (Table 1). A post hoc analysis indicated that this effect was marginally driven by the DSI group showing lower FIM scores than the NSI group ( $p<$ 0.09 ). The HI only group had a pattern of largest functional improvement between admission and discharge, but this effect was not statistically significant $(F(3,58)=0.49, p>$ 0.05 ). As a whole, all patient groups demonstrated functional improvement over time (with increased FIM scores), but no group distinguished itself from any other at a statistically significant level.

\section{Motor Functional Independence Measure Subscales}

At admission, no significant differences were observed between any of the groups. At discharge, the DSI group consistently presented with lower scores in all four subcomponents of the motor FIM (self-care, mobility, locomotion, and sphincter control); however, the group mean values differed significantly only in the self-care subcomponent $(F(3,58)=2.93, p<0.05)$. A post hoc comparison indicated that this effect was marginally driven by the DSI group having poorer self-care than the patients with NSI $(p<0.09)$. Although we observed improvements in all motor FIM scores in all patients over time, we found no significant differences between groups as reflected in their score changes $(p>0.05)$.

\section{Cognitive Functional Independence Measure Subscales}

At admission, we found no clear trend to distinguish the groups' communication and social-cognition scores.
At discharge, while no statistically significant difference between groups existed, the DSI group trended slightly lower on both cognitive subcomponents. Although all groups showed some level of improvement between admission and discharge, we found no statistically significant differences between groups on the cognitive FIM score changes $(p>0.05)$.

\section{Traumatic Brain Injury Severity}

Table 2 shows the distribution of TBI severity stratified by sensory impairment group. Cases of mild and severe TBI occurred equally ( $n=25$ in each category) and slightly more than twice as frequently as cases of moderate TBI $(n=12)$.

\section{Regression Analyses}

Multiple regression analyses were performed to determine significant predictors of total, motor, and cognitive functional change as measured by the FIM gain between admission and discharge. Table 3 shows that the total FIM score at admission was a significant negative predictor of total, motor, and cognitive FIM score changes. In addition, DSI significantly contributed to reduced total and motor

Table 2.

Traumatic brain injury (TBI) severity stratified by sensory impairment group.

\begin{tabular}{lccc}
\hline \multicolumn{1}{c}{$\begin{array}{c}\text { Sensory } \\
\text { Impairment }\end{array}$} & \multicolumn{3}{c}{ TBI Severity } \\
\cline { 2 - 4 } & $\begin{array}{c}\text { Mild } \\
(\boldsymbol{n}=\mathbf{2 5})\end{array}$ & $\begin{array}{c}\text { Moderate } \\
(\boldsymbol{n}=\mathbf{1 2})\end{array}$ & $\begin{array}{c}\text { Severe } \\
(\boldsymbol{n}=\mathbf{2 5})\end{array}$ \\
\hline NSI $(n=9)$ & 5 & 2 & 2 \\
HI Only $(n=12)$ & 1 & 4 & 7 \\
VI Only $(n=21)$ & 10 & 4 & 7 \\
DSI $(n=20)$ & 9 & 2 & 9 \\
\hline DSI $=$
\end{tabular}

DSI = dual sensory impairment, $\mathrm{HI}=$ hearing impairment, $\mathrm{NSI}=$ no sensory impairment, $\mathrm{VI}=$ vision impairment. 
JRRD, Volume 46, Number 6, 2009

Table 3.

Predictors of improvement in Functional Independence Measure (FIM) scores.

\begin{tabular}{|c|c|c|c|c|c|}
\hline Scores & $\begin{array}{l}F \text {-Value } \\
\text { of Model }\end{array}$ & $\begin{array}{c}\text { Adjusted } R^{2} \\
\text { of Model }\end{array}$ & $\begin{array}{l}t \text {-Value of } \\
\text { Predictor }\end{array}$ & $\begin{array}{c}\text { Partial } \\
\text { Correlation } \\
\text { of Predictor }\end{array}$ & $\begin{array}{c}\text { Part } \\
\text { Correlation } \\
\text { of Predictor }\end{array}$ \\
\hline Total FIM Change Model & $51.22 *$ & 0.85 & & & \\
\hline Admission FIM & & & $-16.38^{*}$ & -0.91 & -0.81 \\
\hline DSI & & & $-2.25^{\dagger}$ & -0.29 & -0.11 \\
\hline Motor FIM Change Model & $49.45^{*}$ & 0.85 & & & \\
\hline Cognitive FIM Change Model & $11.65^{*}$ & 0.55 & & & \\
\hline Admission FIM & & & $-7.27^{*}$ & -0.70 & -0.63 \\
\hline DSI & & & -0.33 & -0.05 & -0.03 \\
\hline \multicolumn{6}{|c|}{$\begin{array}{l}\text { Note: Only significant predictors are presented. Nonsignificant predictor variables included in model were vision impairment only, hearing impairment only, age, } \\
\text { time since injury, and traumatic brain injury severity. } \\
{ }^{*} p<0.01 \text {. } \\
{ }^{t} p<0.05 \text {. } \\
\text { DSI = dual sensory impairment. }\end{array}$} \\
\hline
\end{tabular}

FIM score changes but did not have a significant effect on cognitive FIM change.

\section{DISCUSSION}

Several limitations of the current study prevent us from making generalizations. First and foremost, this is a preliminary study with a small sample size $(n=62)$. Moreover, not all of the 175 PRC patients received both the requested examinations, nor did the participants receive the same intensity of rehabilitation because of missed appointments, short lengths of stay, or unresponsiveness due to severity of injury. In addition, although the vision evaluation involved both peripheral and central components, the audiology evaluation did not assess central auditory function. This lack of central auditory testing may account for the low percentage of patients in the HI group. Central auditory testing should be included in follow-up studies.

However, our data did show a higher occurrence of DSI in patients with blast-related TBI (32\%) than in a non-TBI VA outpatient sample (who also had both hearing and vision examinations completed) [15]. The observation of DSI reported in Smith et al.'s patient sample with non-TBI ranged from 0 ( $<65$ years of age) to 22 percent ( $>85$ years of age), with an overall prevalence of 7.4 percent [15]. Their finding suggested that DSI was typically not seen in the traditional VA population until they were $>65$ years old. Yet in the present cohort with an average age of 27 years, 32 percent of the patients had been diagnosed with DSI. This finding may suggest an alarming trend in this new generation of veterans; i.e., premature deterioration in auditory and visual function that will likely have long-term consequences.

The ANOVA between the dependent variable of FIM score and independent measures of HI only, VI only, DSI, and NSI did not reveal any statistically significant findings. We hypothesize that this was because of the relatively small group sizes; therefore, our ANOVA lacked sufficient power. Future studies with larger samples are needed to more fully explore the potential mean differences between groups and functional outcome. We performed a regression model analysis to determine which factors significantly contributed to functional rehabilitation outcome. This analysis demonstrated a strong inverse relationship between function at admission and overall functional improvement. This pattern is frequently observed in the general inpatient rehabilitation population; patients with relatively high levels of functionality at admission may have fewer new tasks to master before they are independent in all categories measured by the FIM. More intriguing, however, we found DSI to negatively predict functional gain during the inpatient stay, indicating that an impairment in both hearing and vision modalities either reduces or impedes functional recovery. Of note, we diagnosed 40 percent of patients with severe TBI. However, the regression analysis demonstrated that level of TBI severity was not a significant predictor of change in FIM scores. This further suggests that the presence of DSI, independent of degree of TBI severity, predicts poorer functional gain. 
We would expect that having HI, VI, or DSI would be detrimental to cognitive performance. However, while DSI negatively predicted gain in the FIM total and motor scores, it did not have an effect on the FIM cognitive subscales of communication and social-cognition. The lack of an effect may be because of FIM's low sensitivity for addressing subtle changes in cognitive function. In follow-up studies, more comprehensive assessment with other established neuropsychological measures may be warranted.

Although not assessed in the current study and not usually categorized as HI, vestibular disturbances are common in patients with TBI and may also contribute to decreased participation in rehabilitation and lesser FIM gains during inpatient stays. By evaluating vestibular symptoms together with auditory and visual symptoms, future studies may provide a more complete view of functional capacities and limitations in this population and lead to the development of rehabilitation techniques that allow clinicians to optimize rehabilitation for DSI patients with TBI. Future studies should also consider the operational definition of the hearing component of DSI. Detailed discussions on the complexity associated with defining DSI are available elsewhere [15].

Previous literature shows that DSI in the geriatric population has been associated with more depressive symptoms [20-21]. In comparison to single sensory impairment, DSI is also associated with lower healthrelated quality of life [22]. Given the relative young age and the complexity of injury of this new veteran population, healthcare systems must anticipate how this cohort will experience multiple functional problems as they age.

Unfortunately, our study did not have enough power to stratify and analyze patients by the mechanism through which the injuries occurred. Also, as a retrospective chart review, our study did not include a control population, raising the question of whether DSI impedes FIM progress in general or just in the population with TBI. Further studies comparing inpatient rehabilitation populations with and without TBI could address this issue.

\section{CONCLUSIONS}

The present study demonstrates that HI and VI are both commonly associated with blast-related TBI. It also suggests that DSI may be detrimental to functional recovery in the rehabilitation process. Our study encourages clinicians caring for patients with TBI to address this issue more promptly and comprehensively. Recently, the VA adopted a policy that all PRC inpatient TBI admissions with sufficient duration will need to receive vision consultation services [23]. Ostensibly, such consultation would also be applied to auditory examinations, because implementation of both examinations should probably be synchronized.

We hope that results from this preliminary study may stimulate additional research efforts and provide insight for clinicians regarding the effect of sensory impairments on TBI rehabilitation.

\section{ACKNOWLEDGMENTS}

\section{Author Contributions:}

Study concept and design: H. L. Lew, G. L. Goodrich. Acquisition of data: D. W. Garvert.

Analysis and interpretation of data: D. W. Garvert, H. L. Lew, G. L. Goodrich, P-T. Hsu, T. K. Pogoda.

Drafting of manuscript: H. L. Lew, D. W. Garvert, T. K. Pogoda, P-T. Hsu, J. M. Devine, D. K. White, P. Myers, G. L. Goodrich.

Critical revision of manuscript for important intellectual content: H. L. Lew, D. W. Garvert, T. K. Pogoda, P-T. Hsu, P. Myers, G. L. Goodrich. Statistical analysis: D. W. Garvert, H. L. Lew, G. L. Goodrich. Obtained funding: H. L. Lew, G. L. Goodrich.

Study supervision: H. L. Lew, G. L. Goodrich.

Financial Disclosures: The authors have declared that no competing interests exist.

Funding/Support: This material was based on work supported in part by a VA Merit Review grant (BR5008) to Dr. Lew and a Quality Enhancement Research Initiative grant (RRP 07-339) to Dr. Goodrich. Additional Contributions: We gratefully acknowledge Dr. Lucille Beck, Dr. David Chandler, Dr. David Cifu, Dr. Barbara Sigford, Dr. Steven Scott, Dr. Joel Scholten, Dr. Sandy Lai, Dr. Micaela Cornis-Pop, Ms. Gretchen Stephens, Ms. Laura Mendelson, Ms. Rose Salerno, Dr. Annabel Castaneda, Ms. Myrlyn Alvarado, and Dr. Lawrence Leung for their support and assistance.

Dr. Lew was at VA Palo Alto Health Care System, Palo Alto, California, during the data acquisition phase of this study. Dr. Devine is now with the Cognitive Neurology Unit, Department of Neurology, Beth Israel Deaconess Medical Center, Boston, Massachusetts.

Participant Follow-Up: The authors do not plan to inform participants of the publication of this article because it was a retrospective study and contact information is unavailable.

\section{REFERENCES}

1. Langlois JA, Rutland-Brown W, Thomas KE. Traumatic brain injury in the United States: Emergency department visits, hospitalizations, and deaths. Atlanta (GA): Centers for Disease Control and Prevention, National Center for Injury Prevention and Control; 2004. 
2. Rutland-Brown W, Langlois JA, Thomas KE, Xi YL. Incidence of traumatic brain injury in the United States, 2003. J Head Trauma Rehabil. 2006;21(6):544-48. [PMID: 17122685] DOI:10.1097/00001199-200611000-00009

3. Hoge CW, McGurk D, Thomas JL, Cox AL, Engel CC, Castro CA. Mild traumatic brain injury in U.S. soldiers returning from Iraq. N Engl J Med. 2008;358(5):453-63. [PMID: 18234750] DOI:10.1056/NEJMoa072972

4. Martin EM, Lu WC, Helmick K, French L, Warden DL. Traumatic brain injuries sustained in the Afghanistan and Iraq wars. Am J Nurs. 2008;108(4):40-47. [PMID: 18367927]

5. Lew HL, Poole JH, Alvarez S, Moore W. Soldiers with occult traumatic brain injury. Am J Phys Med Rehabil. 2005;84(6):393-98. [PMID: 15905652]

DOI:10.1097/01.phm.0000163703.91647.a7

6. Okie S. Traumatic brain injury in the war zone. N Engl J Med. 2005;352(20):2043-47. [PMID: 15901856] DOI:10.1056/NEJMp058102

7. Lew HL, Poole JH, Vanderploeg RD, Goodrich GL, Dekelboum S, Guillory SB, Sigford B, Cifu DX. Program development and defining characteristics of returning military in a VA Polytrauma Network Site. J Rehabil Res Dev. 2007; 44(7):1027-34. [PMID: 18075959]

DOI:10.1682/JRRD.2007.05.0073

8. Madigan NK, DeLuca J, Diamond BJ, Tramontano G, Averill A. Speed of information processing in traumatic brain injury: Modality-specific factors. J Head Trauma Rehabil. 2000;15(3):943-56. [PMID: 10785624] DOI:10.1097/00001199-200006000-00007

9. Myers PJ, Wilmington DJ, Gallum FJ, Henry JA, Fausti SA. Hearing impairment and traumatic brain injury among soldiers: Special considerations for the audiologist. Semin Hear. 2009;30(1):5-27. DOI:10.1055/s-0028-1111103

10. Jury MA, Flynn MC. Auditory and vestibular sequelae to traumatic brain injury: A pilot study. N Z Med J. 2001; 114(1134):286-88. [PMID: 11480511]

11. Lew HL, Jerger JF, Guillory SB, Henry JA. Auditory dysfunction in traumatic brain injury. J Rehabil Res Dev. 2007; 44(7):921-28. [PMID: 18075949]

DOI:10.1682/JRRD.2007.09.0140

12. DePalma RG, Burris DG, Champion HR, Hodgson MJ. Blast injuries. N Engl J Med. 2005;352(13):1335-42.

[PMID: 15800229] DOI:10.1056/NEJMra042083

13. Goodrich GL, Kirby J, Cockerham G, Ingalla SP, Lew HL. Visual function in patients of a polytrauma rehabilitation center: A descriptive study. J Rehabil Res Dev. 2007;44(7): 929-36. [PMID: 18075950]

DOI:10.1682/JRRD.2007.01.0003

14. Wong TY, Seet MB, Ang CL. Eye injuries in twentieth century warfare: A historical perspective. Surv Ophthalmol. 1997;41(6):433-59. [PMID: 9220567] DOI:10.1016/S0039-6257(97)00022-2

15. Smith SL, Bennett LW, Wilson RH. Prevalence and characteristics of dual sensory impairment (hearing and vision) in a veteran population. J Rehabil Res Dev. 2008;45(4):597-609. [PMID: 18712645] DOI:10.1682/JRRD.2007.02.0023

16. Carhart R, Jerger JF. Preferred method for clinical determination of pure tone thresholds. J Speech Hear Disord. 1959; 24:330-45.

17. Martin FN. Introduction to audiology. 3rd ed. Englewood Cliffs (NJ): Prentice-Hall; 1986.

18. International Classification of Diseases-9th Revision. Atlanta (GA): Centers for Disease Control and Prevention.

19. Corrigan JD, Smith-Knapp K, Granger CV. Validity of the functional independence measure for persons with traumatic brain injury. Arch Phys Med Rehabil. 1997;78(8): 828-34. [PMID: 9344301] DOI:10.1016/S0003-9993(97)90195-7

20. Capella-McDonnall ME. The effects of single and dual sensory loss on symptoms of depression in the elderly. Int $\mathrm{J}$ Geriatr Psychiatry. 2005;20(9):855-61. [PMID: 16116571] DOI:10.1002/gps.1368

21. Lupsakko T, Mäntyjärvi M, Kautiainen H, Sulkava R. Combined hearing and visual impairment and depression in a population aged 75 years and older. Int J Geriatr Psychiatry. 2002;17(9):808-13. [PMID: 12221653] DOI:10.1002/gps.689

22. Chia EM, Mitchell P, Rochtchina E, Foran S, Golding M, Wang JJ. Association between vision and hearing impairments and their combined effects on quality of life. Arch Ophthalmol. 2006;124(10):1465-70. [PMID: 17030715] DOI:10.1001/archopht.124.10.1465

23. Veterans Health Administration. Performance of traumatic brain injury specific ocular health and visual functioning examinations for polytrauma rehabilitation center patients. VHA Directive 2008-065. Washington (DC): Department of Veterans Affairs; 2008.

Submitted for publication September 18, 2008. Accepted in revised form August 4, 2009. 